\title{
On the Nonrelativistic Limit of the Dirac Theory
}

\author{
W. Hunziker \\ Institut für Theoretische Physik der ETH, Zürich, Switzerland
}

Received October 15, 1974

\begin{abstract}
The relation between a "nonrelativistic" Hamiltonian of the form $H^{\infty}=(A+B)^{2}+C$ and a corresponding family of "Dirac-Hamiltonians" $H(c)$ in the limit $c \rightarrow \infty$ is investigated. It is shown that the resolvent $(z-H(c))^{-1}$ and the relativistic perturbation of isolated eigenvalues of $H^{\infty}$ are analytic in $1 / c$ for sufficiently large $|c|$.
\end{abstract}

\section{Introduction}

The Hamiltonian of a Dirac-electron of charge $e=1$ and mass $m=1 / 2$ may be written as

$$
H(c)=c \alpha(\boldsymbol{p}-\boldsymbol{A}(\boldsymbol{x}))+\frac{1}{2} \beta c^{2}+\varphi(\boldsymbol{x}),
$$

where $p=-i d / d \boldsymbol{x}$ and with the $4 \times 4$-matrices

$$
\alpha=\left(\begin{array}{ll}
0 & \boldsymbol{\sigma} \\
\boldsymbol{\sigma} & 0
\end{array}\right), \quad \beta=\left(\begin{array}{ll}
\mathbb{1} & 0 \\
0 & \mathbb{1}
\end{array}\right),
$$

whose elements are the $2 \times 2$-matrices $\mathbb{1}$ and $\sigma=\left(\sigma_{1}, \sigma_{2}, \sigma_{3}\right)=$ set of Pauli spinmatrices. $\boldsymbol{A}(\boldsymbol{x})$ and $\varphi(\boldsymbol{x})$ are the potentials of the static electromagnetic field. The usual factor $1 / c$ in front of $\boldsymbol{A}(\boldsymbol{x})$ is omitted on purpose since it must be kept fixed in the nonrelativistic limit $c \rightarrow \infty$. $H(c)$ acts on the Hilbertspace $C^{4} \otimes L^{2}\left(R^{3}\right)$ of square-integrable 4-component wave functions.

On a formal level, it is well understood that the nonrelativistic limit $c \rightarrow \infty$ is described by the Pauli-Hamiltonian

$$
H^{\infty}=\left(\boldsymbol{\sigma}(\boldsymbol{p}-\boldsymbol{A}(\boldsymbol{x}))^{2}\right)+\varphi(\boldsymbol{x})
$$

on the smaller Hilbertspace $C^{2} \otimes L^{2}\left(R^{3}\right)$, and there exists a sytematic scheme for obtaining corrections to $H^{\infty}$ in the form of a power series in $1 / c$ [1]. However, these "relativistic perturbations" of $H^{\infty}$ are given by more and more singular operators which are by no means small with respect to $H^{\infty}$. One might therefore suspect that perturbation expansions in powers of $1 / c$ are at best asymptotic.

Nevertheless, Titchmarsh [2] has proved analyticity in $1 / c$ of eigenvalues and eigenfunctions for the spherically symmetric case without magnetic field: $\varphi=\varphi(r), \boldsymbol{A}=0$; and Veselic [3] has extended this result to the case without spherical symmetry: $\varphi=\varphi(\boldsymbol{x}), \boldsymbol{A}=0$.

In this note we investigate the general case $A \neq 0$ which poses essentially new problems-already in the nonrelativistic limit. One of the points we wish to make is that it is profitable to treat a general Hamiltonian of type $H^{\infty}=(A+B)^{2}+\mathrm{C}$ as a nonrelativistic limit of a corresponding Dirac-Hamiltonian $H(c)$. 
In order to keep the conditions on $\boldsymbol{A}$ and $\varphi$ fairly general, we here restrict ourselves to the discrete spectrum of $H$. The analyticity properties of the resolvent in $1 / c$ will of course also be needed in the discussion of the continuum. However, additional assumptions for the electromagnetic field (like dilatation-analyticity or sufficiently rapid fall-off at infinity) are then necessary and the arguments become more technical [4].

\section{The Hamiltonian and Its Spectrum}

We summarize some (but not all!) known results on the selfadjointness and on the spectrum of $H(c)$. This is intended only as a background for the more general set-up introduced in Section 3.

$H$ is of the form $H_{0}+V$ with $H_{0}=c \boldsymbol{\alpha} \boldsymbol{p}+\frac{1}{2} c^{2} \beta, V(\boldsymbol{x})=\varphi(\boldsymbol{x})-c \boldsymbol{\alpha} \boldsymbol{A}(\boldsymbol{x}) . V(\boldsymbol{x})$ is a $4 \times 4$-matrixvalued function on $R^{3} \cdot L^{p}$-norms of $V$ may be defined with respect to any matrix-norm.

Theorem 1. Let $V \in L^{p}+L^{\infty}$ for some $p>3$. Then $V$ is $H_{0}$-bounded with arbitrarily small relative bound. Therefore, $H=H_{0}+V$ is selfadjoint with domain $D\left(H_{0}\right)$.

Theorem 2. Suppose that $V \in L^{p}+\varepsilon L^{\infty}(p>3)$, i.e. that the $L^{\infty}$-part of $V$ can be chosen arbitrarily small in $L^{\infty}$-norm. Then the spectrum $\sigma(H)$ of $H$ consists of the continuum $\sigma\left(H_{0}\right)=\left\{z \in R:|z| \geqq \frac{1}{2} c^{2}\right\}$ and, in the complement of $\sigma\left(H_{0}\right)$, of isolated eigenvalues with finite multiplicities with can accumulate only at $+\frac{1}{2} c^{2}$.

Remarks. Theorem 1 is proved in [5] and follows from the fact that an operator of the form

$$
f(x)\left(1+p^{2}\right)^{-1 / 2}
$$

on $L^{2}\left(R^{3}\right)$ is bounded if $f \in L^{p}, p>3$. The condition $p>3$ excludes Coulomb-like singularities. However, $f(\boldsymbol{x})=|\boldsymbol{x}|^{-1}$ is still relatively bounded with respect to $|\boldsymbol{p}|[6]$.

Theorem 2 can be proved like its analogue for Schrödinger Hamiltonians [7]. The main point is that (3) is a compact operator if $f \in L^{p}+\varepsilon L^{\infty}$, i.e. $V$ is relatively compact with respect to $H_{0}$.

\section{The Nonrelativistic Limit}

We now pose the problem in a generalized form. Let $A, B, C$ be symmetric operators on a Hilbertspace $\mathscr{H}$. On $C^{2} \otimes \mathscr{H}$ we define the "Dirac-Hamiltonian"

where

$$
H(c)=c \alpha \otimes(A+B)+\frac{c^{2}}{2} \beta \otimes \mathbb{1}+\mathbb{1} \otimes C,
$$

$$
\alpha=\left(\begin{array}{ll}
0 & 1 \\
1 & 0
\end{array}\right), \quad \beta=\left(\begin{array}{rr}
1 & 0 \\
0 & -1
\end{array}\right) .
$$

We want to show that the limit $c \rightarrow \infty$ is described by the "Pauli-Hamiltonian"

$$
H^{\infty}=(A+B)^{2}+C \text { on } \mathscr{H} \text {. }
$$


Corresponding to the hypothesis of Theorem 1 we assume:

$$
\begin{aligned}
& A=A^{*} ; B \text { and } C \text { are } A \text {-bounded, in particular } \\
& B \text { has relative bound }<1 \text { with respect to } A .
\end{aligned}
$$

It follows easily that $H(c)$ is selfadjoint with domain $D(A)$ for $c$ real and sufficiently large. For $H^{\infty}$ we have

Lemma 1. $H^{\infty}$ is selfadjoint with domain $D\left((A+B)^{2}\right)$ and bounded below.

Proof. By (4), $A+B$ is selfadjoint with domain $D(A)$ and $A$ is $(A+B)$-bounded. Hence $C$ is $(A+B)$-bounded and has therefore arbitrarily small relative bound with respect to $(A+B)^{2}$.

Remark. For $H^{\infty}$, the splitting

$$
H^{\infty}=A^{2}+\left(A B+B A+B^{2}+C\right)
$$

into unperturbed part plus perturbation is artificial and raises unneccessary domain questions. These will be avoided automatically by treating $H^{\infty}$ as a limit of $H(c)$.

We first discuss the unperturbed resolvent $\left(z-H_{0}(c)\right)^{-1}$ for

$$
H_{0}(c)=c \alpha A+\frac{1}{2} c^{2} \beta,
$$

where we have dropped the tensor-product notation. From $\alpha^{2}=\beta^{2}=1$ and $\alpha \beta+\beta \alpha=0$ it follows that

$$
H_{0}^{2}(c)=c^{2} A^{2}+\frac{1}{4} c^{4},
$$

which shows that $\sigma\left(H_{0}\right)$ has at least the gap $\left(-\frac{1}{2} c^{2},+\frac{1}{2} c^{2}\right)$. For $z \notin \sigma\left(H_{0}\right)$ we have

$$
\begin{aligned}
\left(z=H_{0}\right)^{-1} & =\left(z+H_{0}\right)\left(z^{2}-H_{0}^{2}\right)^{-1} \\
& =\left(z+c \alpha A+\frac{1}{2} c^{2} \beta\right)\left(z^{2}-\frac{1}{4} c^{4}-c^{2} A^{2}\right)^{-1} .
\end{aligned}
$$

Before taking the nonrelativistic limit $c \rightarrow \infty$ we must subtract from $H_{0}(c)$ or $H(c)$ the rest energy $\frac{1}{2} c^{2}$ or, equivalently, replace in the resolvents $z$ by $z+\frac{1}{2} c^{2}$. This will always be assumed in the following. It is also convenient to use 2-component notation: $u=\left(\begin{array}{l}u^{1} \\ u_{2}\end{array}\right), u_{k} \in \mathscr{H}$, for vectors $u \in C^{2} \otimes \mathscr{H}$ and the corresponding $2 \times 2$-matrix notation for operators on $C^{2} \otimes \mathscr{H}$. The unperturbed resolvent then takes the form

$$
\begin{aligned}
G_{0}(z, c) & =\left(z-H_{0}(c)\right)^{-1} \\
& =\left(\begin{array}{cc}
1+\frac{z}{c^{2}} & \frac{A}{c} \\
\frac{A}{c} & \frac{z}{c^{2}}
\end{array}\right)\left(z+\frac{z^{2}}{c^{2}}-A^{2}\right)^{-1},
\end{aligned}
$$

which shows explicitly that, for $z \notin \sigma\left(A^{2}\right), G_{0}(z, c)$ is analytic in $1 / c$ in a $z$-dependent neighbourhood of $1 / c=0$. To construct the full resolvent $G(z, c)=(z-H(c))^{-1}$ we 
start from the resolvent equation

where

$$
G(z, c)=G_{0}(z, c)+K(z, c) G(z, c),
$$

$$
\begin{aligned}
K(z, c) & =G_{0}(z, c)\left(H(c)-H_{0}(c)\right) \\
& =\left(z+\frac{z^{2}}{c^{2}}-A^{2}\right)^{-1}\left(\begin{array}{cc}
\left(1+\frac{z}{c^{2}}\right) C+A B & \left(1+\frac{z}{c^{2}}\right) c B+\frac{A C}{c} \\
\frac{1}{c}(A C+z B) & A B+\frac{z C}{c^{2}}
\end{array}\right) .
\end{aligned}
$$

This expression for $K$ must be understood in the following sense: a term like $\left(z+\frac{\mathrm{z}^{2}}{c^{2}}-A^{2}\right)^{-1} A B$ is defined on $D(B)$ as the product of the bounded operator $A\left(z+\frac{z^{2}}{c^{2}}-A^{2}\right)^{-1}$ with $B$. As a consequence of (4), $K$ is therefore defined on $D(A)$ and bounded. In the following we denote with $K$ the unique bounded extension of this operator to all of $C^{2} \otimes \mathscr{H}$. For $B \neq 0$ we see that $K$ diverges as $c \rightarrow \infty$. To control this divergence we set

and introduce

$$
S(c)=\left(\begin{array}{ll}
1 & 0 \\
0 & c
\end{array}\right)
$$

$$
\begin{aligned}
& \tilde{G}_{0}(z, c)=S G_{0} S^{-1}=\left(z+\frac{z^{2}}{c^{2}}-A^{2}\right)^{-1}\left(\begin{array}{cc}
1+\frac{z}{c^{2}} & \frac{A}{c^{2}} \\
A & \frac{z}{c^{2}}
\end{array}\right) \\
& =\left(z-A^{2}\right)^{-1}\left(\begin{array}{ll}
1 & 0 \\
A & 0
\end{array}\right) \text { for } c=\infty \text {. } \\
& \tilde{K}(z, c)=S K S^{-1}=\left(z+\frac{z^{2}}{c^{2}}-A^{2}\right)^{-1}\left(\begin{array}{cc}
\left(1+\frac{z}{c^{2}}\right) C+A B & \left(1+\frac{z}{c^{2}}\right) B+\frac{A C}{c^{2}} \\
A C+z B & A B+\frac{z C}{c^{2}}
\end{array}\right) \\
& =\left(z-A^{2}\right)^{-1}\left(\begin{array}{cc}
C+A B & B \\
A C+z B & A B
\end{array}\right) \text { for } c=\infty .
\end{aligned}
$$

We notice that for $z \notin \sigma\left(A^{2}\right), \tilde{G}_{0}(\mathrm{z}, \mathrm{c})$ and $\tilde{K}(z, c)$ are analytic in $(1 / c)^{2}$ in a neighbourhood of zero. The resolvent equation transforms into

$$
\tilde{G}(z, c)=\tilde{G}_{0}(z, c)+\tilde{K}(z, c) \tilde{G}(z, c)
$$

for $\hat{G}=S G S^{-1}$. Our next task is to connect this equation for $c=\infty$ with the Pauli Hamiltonian $H^{\infty}$. 
Theorem 3. Let $A, B, C$ satisfy (4) and let $z \notin \sigma\left(A^{2}\right)$. Then the two equations

$$
\left(z-H^{\infty}\right) u=v
$$

and

$$
\left(\begin{array}{l}
u_{1} \\
u_{2}
\end{array}\right)=\left(\begin{array}{c}
\left(z-A^{2}\right)^{-1} v \\
A\left(z-A^{2}\right)^{-1} v
\end{array}\right)+\tilde{K}(z, \infty)\left(\begin{array}{l}
u_{1} \\
u_{2}
\end{array}\right)
$$

are equivalent in the following sense: (8) implies (9) for $\left(\begin{array}{l}u_{1} \\ u_{2}\end{array}\right)=\left(\begin{array}{c}u \\ (A+B) u\end{array}\right)$,(9) implies $u_{1} \in D\left((A+B)^{2}\right), u_{2}=(A+B) u_{1}$ and (8) for $u=u_{1}$.

The straightforward but somewhat lengthy proof is given in Section 5. As a corollary we note that for $z \notin \sigma\left(A^{2}\right), z \notin \sigma\left(H^{\infty}\right), G^{\infty}(z)=\left(z-H^{\infty}\right)^{-1}$ satisfies

$$
\left(\begin{array}{cc}
G^{\infty}(z) & 0 \\
(A+B) G^{\infty}(z) & 0
\end{array}\right)=\left(\begin{array}{cc}
\left(z-A^{2}\right)^{-1} & 0 \\
A\left(z-A^{2}\right)^{-1} & 0
\end{array}\right)+\tilde{K}(z, \infty)\left(\begin{array}{cc}
G^{\infty}(z) & 0 \\
(A+B) G^{\infty}(z) & 0
\end{array}\right)
$$

Conversely, if $z \notin \sigma\left(A^{2}\right)$ and if $(1-\tilde{K}(z, \infty))^{-1}$ exists and is bounded, it follows that $z \notin \sigma\left(H^{\infty}\right)$ and that

$$
\left(\begin{array}{cc}
G^{\infty}(z) & 0 \\
(A+B) G^{\infty}(z) & 0
\end{array}\right)
$$

is the unique solution of $(10)$ in $L\left(C^{2} \otimes \mathscr{H}\right)$. Therefore, (10) is a suitable resolvent equation for $H^{\infty}$ which may also be used, incidentally, as a starting point for timeindependent scattering theory [4]. Corresponding to the hypothesis of theorem 2 we now assume in addition to (4) that

$$
B \text { and } C \text { are relatively compact with respect to } A \text {. }
$$

As in Theorem 2 it then follows that $H(c)$ can only have isolated eigenvalues of finite multiplicities in the complement of $\sigma\left(H_{0}(c)\right)$, in particular in the gap $\left(-c^{2}, 0\right)$ (rest energy subtracted). Since $\tilde{K}(z, \infty)$ is compact for $z \notin \sigma\left(A^{2}\right)$ we obtain a similar result for $H^{\infty}$ :

Lemma 2. In the complement of $\sigma\left(A^{2}\right), \sigma\left(H^{\infty}\right)$ consists only of isolated eigenvalues of finite multiplicities which are bounded below.

Proof. Suppose that for some $z \notin \sigma\left(A^{2}\right)$, the homogeneous equation

$$
\tilde{K}(z, \infty) u=u
$$

has a nontrivial solution. Then $u=\left(\begin{array}{l}u_{1} \\ u_{2}\end{array}\right)$ satisfies (9) with $v=0$. By Theorem 3, it follows that $u_{1} \in D\left((A+B)^{2}\right), u_{2}=(A+B) u_{1}$ and

$$
\left(z-H^{\infty}\right) u_{1}=0 .
$$

We conclude that $u_{1} \neq 0$ and that $z \in \sigma\left(H^{\infty}\right)$. By the Fredholm alternative, therefore, $(1-\tilde{K}(z, \infty))^{-1}$ exists for $z \notin \sigma\left(H^{\infty}\right)$. Since $\tilde{K}(z, \infty)$ is analytic in $z \notin \sigma\left(A^{2}\right)$, 
$(1-\tilde{K}(z, \infty))^{-1}$ and therefore $G^{\infty}(z)$ are meromorphic in $z \notin \sigma\left(A^{2}\right)$. Conversely, (13) implies (12) for $u=\left(\begin{array}{c}u_{1} \\ (A+B) u_{1}\end{array}\right)$, hence the eigenvalues of $H^{\infty}$ in the complement of $\sigma\left(A^{2}\right)$ are of finite multiplicity. Boundedness below is obvious from Lemma 1. This concludes the proof.

We are now prepared to discuss the analyticity properties in $1 / c$ of $G(z, c)$. Let $z \notin \sigma\left(A^{2}\right) \cup \sigma\left(H^{\infty}\right)$. Then $(1-\tilde{K}(z, \infty))^{-1}$ exists. Since $\tilde{K}(z, c)$ is analytic in $(1 / c)^{2}$ it follows that $(1-\tilde{K}(z, c))^{-1}$ exists and is analytic in $(1 / c)^{2}$ for $|c|$ sufficiently large. The same is true for $\widetilde{G}_{0}(z, c)$ and therefore, by $(7)$, for $\tilde{G}(z, c)$. From $(5)$ and $(10)$ we see that the power series of $\widetilde{G}(z, c)$ in $(1 / c)^{2}$ begins with

$$
\tilde{G}(z, c)=\left(\begin{array}{cc}
G^{\infty}(z) & 0 \\
(A+B) G^{\infty}(z) & 0
\end{array}\right)+0\left(\frac{1}{c^{2}}\right) .
$$

Due to the particular form of the leading term, $G(z, c)=S^{-1}(c) \tilde{G}(z, c) S(c)$ is still analytic in $1 / c$ with an expansion

$$
G(z, c)=\left(\begin{array}{cc}
G^{\infty}(z) & 0 \\
0 & 0
\end{array}\right)+\frac{1}{c}\left(\begin{array}{cc}
0 & (A+B) G^{\infty}(z) \\
(A+B) G^{\infty}(z) & 0
\end{array}\right)+0\left(\frac{1}{c^{2}}\right) .
$$

In general, the diagonal elements of $G(z, c)$ are even in $1 / c$, the off-diagonal elements are odd. These analyticity properties of the resolvent are the basis from which the analyticity properties of eigenvalues and eigenfunctions follow in the usual way [6]. As has been remarked by Veselić, the fact that $G(z, \infty)$ is a pseudoresolvent rather than a resolvent is thereby no obstacle. We only give the final result:

Theorem 4. Let $A, B, C$ satisfy (4) and (11). Let $z$ be an eigenvalue of $H^{\infty}$ in the complement of $\sigma\left(A^{2}\right)$ and $m$ its (finite) multiplicity. Then $z$ is the limit for $c \rightarrow \infty$ of eigenvalues $z_{k}(c)$ of $H(c)$ (with rest-energy subtracted) of total multiplicity $\mathrm{m}$. The functions $z_{k}(c)$ are analytic in $(1 / c)^{2}$ for $|c|$ sufficiently large. An orthonormal set of corresponding eigenvectors of $H(c)$ can be chosen such that each eigenvector is of the form $\left(\begin{array}{l}u_{1}(c) \\ u_{2}(c)\end{array}\right)$ where $u_{1}(c)$ and $c^{-1} u_{2}(c)$ are analytic in $(1 / c)^{2}$ and where $u_{1}(\infty)$ is an eigenvector of $H^{\infty}$ with eigenvalue $z$.

Remark. In general there will be other eigenvalues of $H(c)$ which for $c \rightarrow \infty$ will not converge to an eigenvalue of $H^{\infty}$ in the complement of $\sigma\left(A^{2}\right)$. However, these eigenvalues will leave any compact not intersecting $\sigma\left(A^{2}\right)$, i.e. they will either join $\sigma\left(A^{2}\right)$ or disappear to $\pm \infty$. In fact it is equally possible to study the limit $c \rightarrow \infty$ of $-H(c)-\frac{1}{2} c^{2}$ which leads to a Pauli Hamiltonian $(A+B)^{2}-C$.

\section{Proof of Theorem 3}

$(8) \rightarrow(9)$ :

We define $u_{1}=u$ and $u_{2} \in D(A)$ by

$$
(A+B) u_{1}-u_{2}=0 \text {. }
$$


Then (8) takes the form

$$
(z-C) u_{1}-(A+B) u_{2}-v=0,
$$

and the combination $A\left(z-A^{2}\right)^{-1}(14)-\left(z-A^{2}\right)^{-1}(15)$ gives the upper component of (9)

$$
u_{1}=\left(z-A^{2}\right)^{-1} v+A\left(z-A^{2}\right)^{-1} B u_{1}+\left(z-A^{2}\right)^{-1} C u_{1}+\left(z-A^{2}\right)^{-1} B u_{2} .
$$

All terms in (16) are in $D(A)$. The combination $A(16)+(14)$ leads to the lower component of (9):

$$
u_{2}=A\left(z-A^{2}\right)^{-1} v+z\left(z-A^{2}\right)^{-1} B u_{1}+A\left(z-A^{2}\right)^{-1} C u_{1}+A\left(z-A^{2}\right)^{-1} B u_{2} \text {. }
$$

$(9) \rightarrow(8)$ :

(9) is equivalent to the set (16) (17) with two important modifications. First, all operator products must be replaced by their bounded extensions to $\mathscr{H}$. To indicate this extension we write, for example, $\left[A\left(z-A^{2}\right)^{-1} B\right]$ for the extension of $A\left(z-A^{2}\right)^{-1} B$. Secondly, we start only with the information that $u_{1}$ and $u_{2}$ are in $\mathscr{H}$. The combination $A\left(z-A^{2}\right)^{-1}(16)+\left(z-A^{2}\right)^{-1}(17)$ gives

$$
\left[\left(z-A^{2}\right)^{-1}(A+B)\right] u_{1}=\left(z-A^{2}\right)^{-1} u_{2},
$$

where we have used identities like

$$
A\left(z-A^{2}\right)^{-1}\left[\left(z-A^{2}\right)^{-1} C\right]=\left(z-A^{2}\right)^{-1}\left[A\left(z-A^{2}\right)^{-1} C\right],
$$

which hold trivially on $D(A)$ and extend by continuity to all of $\mathscr{H}$. We now take the scalar product of (18) with an arbitrary $f \in \mathscr{H}$ and set $g=\left(\bar{z}-A^{2}\right)^{-1} f$. Using $\left[\left(z-A^{2}\right)^{-1}(A+B)\right]^{*}=(A+B)\left(\bar{z}-A^{2}\right)^{-1}$ we find

$$
\left((A+B) g, u_{1}\right)=\left(g, u_{2}\right)
$$

for all $g \in D\left(A^{2}\right)$. Since $A$ is the closure of its restriction to $D\left(A^{2}\right)$ and since $B$ is $A$-bounded, this extends by continuity to all $g \in D(A)$. Since $A+B$ is selfadjoint with domain $D(A)$, it follows that

$$
u_{1} \in D(A+B) \text { and } u_{2}=(A+B) u_{1} .
$$

Writing (20) in the form

$$
u_{1}=z\left(z-A^{2}\right)^{-1} u_{1}+A\left(z-A^{2}\right)^{-1} B u_{1}-A\left(z-A^{2}\right)^{-1} u_{2}
$$

and subtracting this from (16) we get

$$
\left(z-A^{2}\right)^{-1}(z-C) u_{1}=\left(z-A^{2}\right)^{-1} v+\left[\left(z-A^{2}\right)^{-1}(A+B)\right] u_{2} .
$$

In the same way as before we conclude that

$$
\left((A+B) g, u_{2}\right)=\left(g,(z-C) u_{1}-v\right)
$$

for all $g \in D(A)$. It follows that $u_{2} \in D(A+B)$ and $(A+B) u_{2}=(z-C) u_{1}-v$. Combined with (20) this is the desired result:

$$
u_{1} \in D\left((A+B)^{2}\right), \quad u_{2}=(A+B) u_{1} \quad \text { and } \quad\left(z-H^{\infty}\right) u_{1}=v
$$




\section{References}

1. See for instance Bjorken, J. D., Drell, S. D. : Relativistic Quantum Mechanics. New York: Mc Graw Hill Inc. 1964

2. Titchmarsh, E. C.: Proc. Roy. Soc. 266 A, 33 (1962)

3. Veselić, K.: Commun. math. Phys. 22, 27 (1971)

4. Hunziker, W.: unpublished report, available from the Institute of theoretical physics of the ETH

5. Prosser, R. T.: J. Math. Phys. 4, 1048 (1964)

6. Kato, T.: Perturbation theory for linear operators. Berlin-Heidelberg-New York: Springer 1966

7. Hunziker, W.: Helv. Phys. Acta 39, 452 (1966)

Communicated by K. Hepp
W. Hunziker

Institut für Theoretische Physik der E.T.H.

CH-8049 Zürich, Switzerland 